\title{
Heterologous expression of PA8FAD9 and functional characterization of a $\Delta 9$-fatty acid desaturase from a cold-tolerant Pseudomonas sp. A8
}

\begin{abstract}
Fatty acid desaturase enzymes are capable of inserting double bonds between carbon atoms of saturated fatty acyl-chains to produce unsaturated fatty acids. A gene coding for a putative $\Delta 9$-fatty acid desaturase-like protein was isolated from a cold-tolerant Pseudomonas sp. A8, cloned and heterologously expressed in Escherichia coli. The gene named as PA8FAD9 has an open reading frame of $1185 \mathrm{bp}$ and codes for 394 amino acids with a predicted molecular weight of $45 \mathrm{kDa}$. The enzyme showed high $\Delta 9$-fatty acid desaturase-like protein activity and increased overall levels of cellular unsaturated fatty acids in the recombinant E. coli cells upon expression at different temperatures. The results showed that the ratio of palmitoleic to palmitic acid in the recombinant E. coli cells increased by more than twice the amount observed in the control cells at $20{ }^{\circ} \mathrm{C}$ using $0.4 \mathrm{mM}$ IPTG. GCMS analysis confirmed the ability of this enzyme to convert exogenous stearic acid to oleic acid incorporated into the recombinant E. coli membrane phospholipids. It may be concluded that the PA8FAD9 gene from Pseudomonas sp. A8 codes for a putative $\Delta 9$-fatty acid desaturase protein actively expressed in E. coli under the influence of temperature and an inducer.
\end{abstract}

Keyword: Pseudomonas sp. A8; $\Delta 9$-fatty acid desaturase; Unsaturated fatty acids; Expression; Membrane phospholipids 